\title{
Leptin and Adiponectin During the First Week After Kidney Transplantation: Biomarkers of Graft Dysfunction?
}

\author{
Isabel Fonseca $^{a, b, c, *}$, José Carlos Oliveira ${ }^{d}$, Josefina Santos ${ }^{a, b}$, Jorge Malheiro ${ }^{a, b}$, \\ La Salete Martins $^{a, b}$, Manuela Almeida ${ }^{a, b}$, Leonídio Dias ${ }^{a}$, Sofia Pedroso ${ }^{a, b}$, \\ Luísa Lobato $^{a, b}$, António Castro Henriques ${ }^{a, b}$, Denisa Mendonça ${ }^{e, c}$ \\ a Department of Nephrology and Kidney Transplantation, Centro Hospitalar do Porto, Hospital de Santo António, Porto, Portugal \\ ${ }^{\mathrm{b}}$ Unit for Multidisciplinary Investigation in Biomedicine (UMIB), Porto, Portugal \\ ${ }^{\mathrm{c}}$ Institute of Public Health (ISPUP), University of Porto, Porto, Portugal \\ d Department of Clinical Chemistry, Centro Hospitalar do Porto, Hospital de Santo António, Porto, Portugal \\ e Department of Population Studies, Institute of Biomedical Sciences Abel Salazar (ICBAS), University of Porto, Porto, Portugal
}

\section{A R T I C L E I N F O}

Article history:

Received 9 July 2014

Accepted 3 October 2014

\section{Keywords:}

Leptin

Adiponectin

Adipokines

Kidney transplantation

Delayed graft function

\section{A B S T R A C T}

Context and Objective. Based on evidence that leptin and adiponectin are removed from circulation primarily by the kidney, we designed a study to examine the longitudinal changes of these adipokines during the first week after kidney transplantation (KTx) and to test the hypothesis that higher levels of leptin and/or adiponectin could be early biomarkers of delayed graft function (DGF = dialysis requirement during the first post-transplant week) and acute rejection.

Study Design. Repeated-measures prospective study.

Material and Methods. Forty consecutive adult patients with end-stage renal disease who were undergoing KTx. Leptin and adiponectin were measured in blood samples that were collected before (day-0) and after KTx (days-1, 2, 4 and 7). Linear mixed-models, receiver operating characteristic and area under curve (AUC-ROC) were used.

Results. At post-transplant day-1, leptinemia and adiponectinemia declined $43 \%$ and $47 \%$, respectively. At all times studied after KTx, the median leptin levels were significantly higher in patients developing DGF $(\mathrm{n}=18)$, but not adiponectin levels. Shortly after KTx (day-1), leptin values were significantly higher in DGF recipients in contrast to patients with promptly functioning kidneys, approximately two times higher when controlling for gender and BMI. The leptin reduction rate between pre-tranplant and one-day after KTx moderately predicted DGF (AUC $=0.73$ ). On day-1, serum leptin predicted DGF (AUC-ROC $=0.76)$ with a performance slightly better than serum creatinine (AUC-ROC $=0.72$ ), even after correcting for BMI (AUC-ROC $=0.73$ ). Separating this analysis by gender showed that the performance of leptin in predicting DGF for male gender (AUC-ROC $=0.86$ ) improved.

Abbreviations: AUC-ROC, Area under the receiver-operating characteristic curve; ADPN, Adiponectin; DGF, Delayed graft function; KTx, Kidney transplantation; SCr, Serum creatinine.

* Corresponding author at: Department of Nephrology and Kidney Transplantation, Centro Hospitalar do Porto, Hospital de Santo António, Largo Prof. Abel Salazar, 4099-001 Porto, Portugal. Tel.: +351 914740001; fax: +351 223320318.

E-mail address: isabelf27@gmail.com (I. Fonseca). 
Conclusions. Kidney graft function is an independent determinant of leptin levels, but not of adiponectin. Leptin levels at day-1 slightly outperformed serum creatinine in predicting the occurrence of DGF, and more accurately in male gender. No significant association was detected with acute rejection.

(c) 2015 Elsevier Inc. All rights reserved.

\section{Introduction}

White adipose tissue is now recognized as a multifunctional organ [1]. In addition to its central role in lipid storage, white adipose tissue has a major endocrine function by synthesizing a multitude of protein cytokines termed adipokines. Leptin and adiponectin (ADPN) are two adipokines that elicit generally opposing pro-inflammatory and anti-inflammatory effects [1-4]. In chronic kidney disease, the clinical significance and prognostic implications of leptin and ADPN are not well understood. Patients with chronic kidney disease have increased circulating levels of both adipokines that may result from an increase in their systemic production and/or decrease in their renal clearance [5-13].

The contribution of the kidney in the biodegradation and elimination of leptin and adiponectin appears to be confirmed in studies conducted in kidney transplantation (KTx), where the circulating levels of these adipokines decrease after successful transplantation [14-19]. Based on the growing evidence that leptin and ADPN are removed from the circulation primarily by the kidney, we designed the present study to examine the longitudinal changes of these two adipokines during the first week post-transplant and test the hypothesis that delayed graft function (DGF) would be associated with higher plasma levels of leptin and due to their impaired clearance by the kidney. Therefore, we hypothesized that higher levels of leptin and/or ADPN could serve as early biomarkers of DGF (defined as dialysis requirement within the first week after KTx). Due to the role of leptin in the immune system [9,20-24], the performance of leptin in predicting acute transplant rejection and alloimmunity was also assessed.

\section{Patients and Methods}

\subsection{Study Design and Patient Population}

Consecutive patients with end-stage renal disease who were undergoing living or deceased donor KTx between December 2010 and May 2011 were prospectively enrolled. Patients under the age of 18 or who required multiorgan transplants were not included. The Institutional Review Board of Centro Hospitalar do Porto approved the study. Each participant provided informed consent.

\subsection{Laboratory Analyses}

Blood samples were collected as follows: $3-6 \mathrm{~h}$ prior to transplant surgery (pre-transplant); on the following morning, approximately 8-12 h after graft reperfusion (day-1); and then on the second (day-2), fourth (day-4) and seventh days (day-7) after transplant, for a total of five samples per patient.
Serum levels of leptin and ADPN were measured by ELISA based on the direct sandwich technique using kits from Mercodia, Sweden. Standard values of leptin for normal weight people were $2-5.6 \mathrm{ng} / \mathrm{mL}$ for male and $3.7-11.1 \mathrm{ng} / \mathrm{mL}$ for female. Expected normal values for ADPN were $5-300 \mathrm{ng} / \mathrm{mL}$.

\subsection{Definitions}

Delayed graft function was defined by the need for dialysis during the first week after KTx.

Acute rejection was defined as either biopsy-proven rejection or anti-rejection treatment without biopsy.

Leptin/BMI ratio was calculated to measure the leptin level while controlling for the BMI contribution.

\subsection{Statistical Analyses}

The distributions of continuous variables were analyzed using Kolmogorov-Smirnov test and variables showing a positively skewed distribution (leptin and $\mathrm{SCr}$ ) were natural logarithm transformed prior to parametric test analyses.

Statistical analysis was conducted in three steps. First, a cross-sectional bivariate analysis was performed. Comparisons of continuous variables between groups were carried out using parametric (t-test) or nonparametric (Mann-Whitney) tests; associations between categorical variables were analyzed using the $x^{2}$ test and Fisher's exact test, as appropriate; correlations were assessed using the Pearson or Spearman correlation.

Second, a linear mixed-effects model was used to evaluate the association of DGF with serial changes of leptin (log-transformed) and ADPN, controlling for the recipient's age, gender and BMI.

Third, a receiver operating characteristic (ROC) curve analysis was performed to assess the utility of the levels of leptin and ADPN (as well as SCr) in predicting DGF. The optimal cut-off points were determined.

Statistical analyses were performed using SPSS version 21.0, and a significance level of 0.05 was considered.

\section{Results}

\subsection{Study Cohort}

The final study cohort included 40 patients. Their demographic and transplant data are shown in supplementary material (Table S1).

\subsection{Leptin and Adiponectin}

The time-course of leptin and ADPN levels during the first post-transplant week is summarized in Table 1. Compared to before the transplant, the median leptin levels declined 
Table 1 - Time course of the circulating levels of leptin (ln) and adiponectin within the first post-transplantation week.

\begin{tabular}{llccccc} 
& & Before KTx & 1st Day & 2nd Day & 4th Day & 7th Day \\
\hline Leptin (ng/mL) & Overall & $15.9[8.6-31.7]$ & $8.4[5.3-20.0]$ & $8.0[3.4-14.7]$ & $8.4[0.8-25.2]$ & $10.5[2.9-25.2]$ \\
Median [IQR] & DGF & $27.4[12.9-57.7]$ & $12.8[7.5-22-6]$ & $13.0[8.7-27.2]$ & $23.1[1.6-36.7]$ & $16.0[9.7-26.8]$ \\
& Non-DGF & $11.6[5.6-41.2]$ & $5.5[2.2-10.0]$ & $4.2[2.4-10.0]$ & $0.8[6-0-15.6]$ & $1.7[5.3-23.1]$ \\
Adiponectin (ng/mL) & Overall & $226(72)$ & $119(35)$ & $114(33)$ & $139(35)$ & $165(29)$ \\
Mean (S.D) & DGF & $250(80)$ & $110(39)$ & $105(37)$ & $130(39)$ & $157(32)$ \\
& Non-DGF & $209(62)$ & $126(30)$ & $120(29)$ & $146(30)$ & $170(25)$ \\
\hline
\end{tabular}

The values shown are the medians and interquartile ranges or the means and standard deviations;

Abbreviations: $\mathrm{KTx}=$ kidney transplantation; DGF = delayed graft function; $\mathrm{SD}$ = standard deviation.

* 1st day $=8-12 \mathrm{~h}$ after surgery.

significantly at day-1, by approximately $47 \%$, but on day- 7 , a less-pronounced reduction of approximately $34 \%$ was observed. With regard to ADPN, compared to before the transplant, the mean levels of plasma ADPN declined by approximately $47 \%$ at day- 1 and $27 \%$ at day- 7 .

As expected, the leptin values were positively correlated with BMI at all time points, whereas no correlation was found with ADPN. Levels of leptin, but not ADPN, were significantly higher in female patients, even after adjustment for BMI, and correlated positively with the recipients' ages at all time points. Neither leptin nor ADPN was significantly correlated with time on dialysis, age of the transplant donor, HLA mismatches and cold ischemia time. Additionally, no significant differences were found in relation to the donor status or any type or dose of the immunosuppressive drugs used.

\subsection{Delayed Graft Function and Acute Rejection}

Eighteen (45\%) patients had DGF, whereas 22 (55\%) patients experienced prompt graft function. Ten recipients had an acute rejection episode during their hospitalization for transplantation, and acute rejection was more frequently diagnosed in patients with DGF than in those with prompt graft function ( $44 \%$ vs. $9 \%, P=0.025)$. Only 2 of the non-DGF patients experienced an acute rejection episode.

3.4. Longitudinal Changes in the Levels of Leptin and Adiponectin According to Graft Function

Prior to transplantation, no significant differences were found between patients with DGF or non-DGF with regard to any of the adipokines evaluated. After transplantation, the mean levels of leptin were consistently higher in DGF patients at all time points compared to non-DGF recipients (Table 1 and Fig. 1), whereas no differences were noted in the mean levels of ADPN.

A linear mixed-effects model was used to analyze the longitudinal changes in leptin and ADPN in the two groups of patients (DGF and non-DGF) by controlling for variables found to be associated by the bivariate analysis (recipient age, gender and BMI) and confirmed the independent association of DGF with the longitudinal changes of leptinemia but not adiponectinemia. Regarding leptin, the age of the recipients was no longer statistically significant and was removed from the final model. The time measurements of leptin, patient
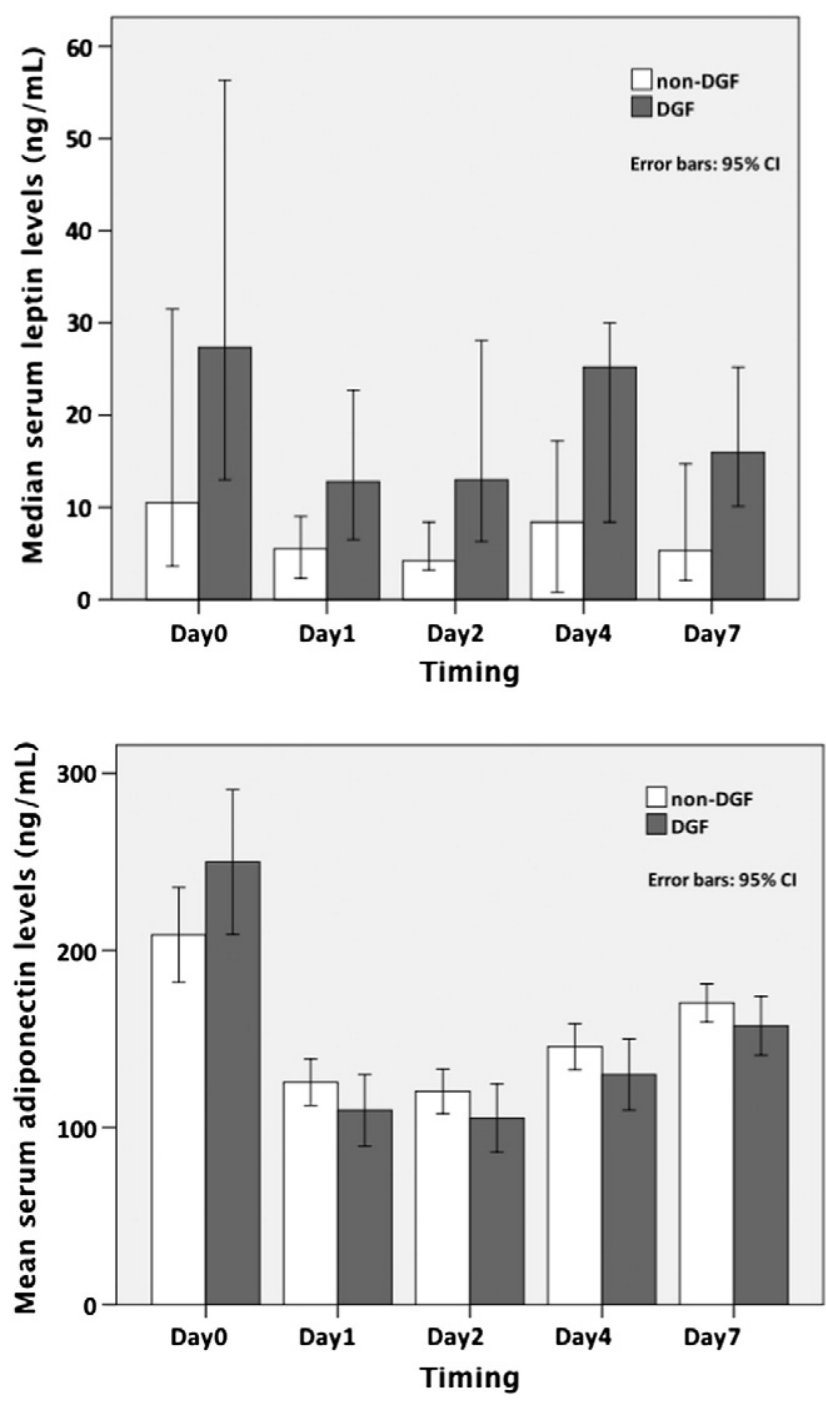

Fig. 1 - Longitudinal changes of the pre-transplant and one-week post-transplant circulating levels of leptin and adiponectin regarding graft function.Evolution of mean and median values of adiponectin and leptin, respectively, with $95 \%$ confidence intervals; measurements were performed preoperatively (day 0), and then at first (day 1), second (day 2), fourth (day 4) and seventh (day 7) days after kidney transplantation. 


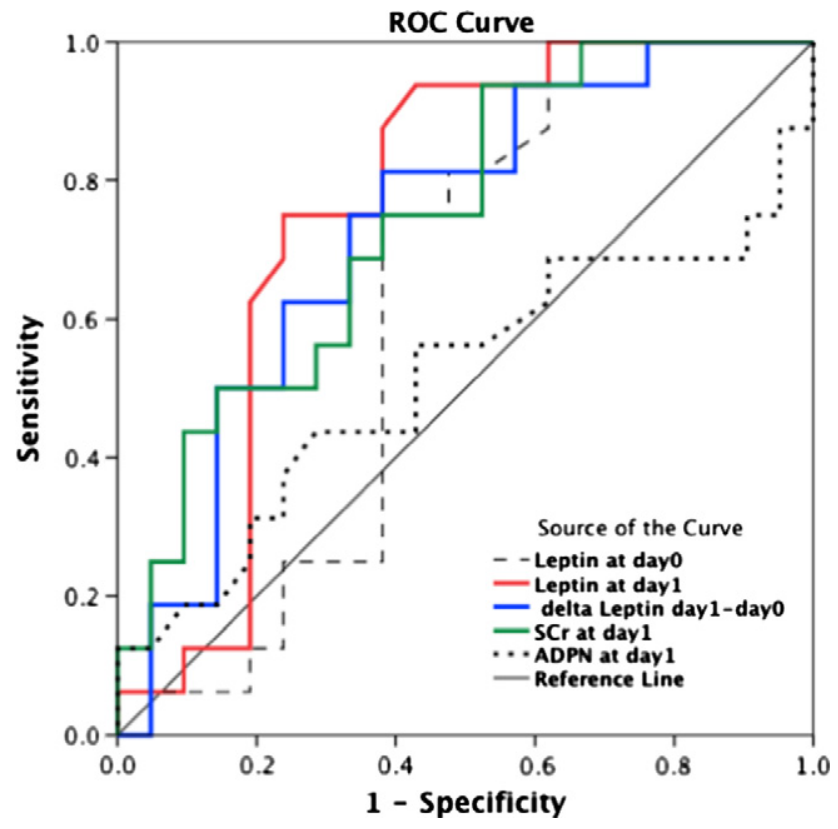

\begin{tabular}{llc}
\hline & AUC $(95 \% \mathrm{Cl})$ & $P$ Value \\
\hline $\begin{array}{l}\text { Day 0 (pre-transplant) } \\
\text { Serum leptin }(\mathrm{ng} / \mathrm{mL})\end{array}$ & $0.62(0.43-0.81)$ & 0.220 \\
\hline Day 1 ( 8 to $12 \mathrm{~h}$ after surgery) & & \\
Serum leptin $(\mathrm{ng} / \mathrm{mL})$ & $0.76(0.60-0.92)$ & 0.008 \\
Serum creatinine $(\mathrm{mg} / \mathrm{dl})$ & $0.72(0.59-0.90)$ & 0.012 \\
Serum adiponectin $(\mathrm{ng} / \mathrm{mL})$ & $0.51(0.31-0.72)$ & 0.676 \\
\hline Leptin reduction rate $(\%)$ & $0.73(0.57-0.90)$ & 0.017 \\
$\Delta$ Leptin $_{\text {day } 1 \text {-dayo }}(\%)$ & & \\
\hline
\end{tabular}

Fig. 2 - Receiver operating characteristic curves for the serum levels of pre-transplant leptin, and leptin, adiponectin and creatinine measured on the first day after KTx, for predicting delayed graft function (DGF). The table lists the areas under the ROC curves of leptin, adiponectin and creatinine for predicting DGF.Abbreviations: $\mathrm{SCr}=$ serum creatinine; ADPN = adiponectin; AUC = area under the ROC curve. $\Delta$ Leptin day1-dayo $=$ percentage changes in serum leptin between day-1 and day- 0 .

gender, BMI and DGF were independent factors associated with leptinemia (Table S2). Even after adjusting for recipient gender and BMI, the DGF remained significantly associated with the longitudinal changes of leptinemia: the recipients with prompt graft function presented lower average leptinemia at all times studied. According to our estimation, the first mean leptin values after transplantation will be approximately two times higher in DGF patients when controlling for recipient gender and BMI. A significant interaction was observed between the recipient gender and $\mathrm{BMI}$, which was retained in the model, meaning that the effect of BMI on leptin changes is different according the gender, i.e., the effect of an increase in BMI is more pronounced in males and attenuated in females.

\subsection{Predicting DGF Using Leptin and Adiponectin}

Receiver operating characteristic (ROC) analysis was conducted to evaluate the performance of leptin and ADPN in discriminating the transplant recipients with DGF. This analysis showed that leptin at day-1 was moderately accurate in predicting the need for dialysis within the first week posttransplant, whereas ADPN was not. The area under the curve (A.UC) for the prediction of DGF was $0.76(P=0.007 ; 95 \% \mathrm{CI}$ : 0.60-0.92) for leptin on day-1, which was slightly better than the diagnostic performance of SCr (AUC $=0.72, P=0.012 ; 95 \%$ CI: 0.59-0.90) (Fig. 2). When adjusted for BMI (leptin/BMI ratio), the performance of one-day leptin in predicting DGF was similar (AUC $=0.73, P=0.017 ; 95 \%$ CI: 0.57-0.90). The potential of pre-transplant leptin levels (day-0) in predicting DGF was also assessed due to higher levels of leptin in DGF vs. non-DGF patients before $\mathrm{KTx}$, but this variable performed poorly having a non-significant AUC of 0.62 .

An ROC curve was also created to evaluate the performance of the reduction in serum leptin levels between pretransplant and day-1 ( $\Delta$ leptin day 1-dayo) in predicting DGF and resulted in an AUC of 0.73 comparable to one-day SCr (Fig. 2). In analyzing the ROC curve of $\Delta$ leptin day 1-dayo, the optimal sensitivity and specificity were achieved at a leptin reduction ratio of $41.7 \%$ (sensitivity: $81 \%$; specificity: $62 \%$; positive and negative predictive values of 68 and 77 , respectively).

In analyzing the ROC curve of leptin on day-1, the optimal sensitivity and specificity were achieved at a leptin concentration of $10.8 \mathrm{ng} / \mathrm{mL}$ (sensitivity: 73\%; specificity: 77\%; positive and negative predictive values of 76 and 74, respectively). Separate analysis by gender, showed that the performance of leptin at day-1 in diagnosing DGF improved considerably for male gender (AUC $=0.86, P=0.004 ; 95 \% \mathrm{CI}$ : $0.70-1.00$ ), and the optimal sensitivity and specificity were achieved at a leptin concentration of $6.4 \mathrm{ng} / \mathrm{mL}$ (sensitivity: $89 \%$; specificity: $79 \%$; positive and negative predictive values of 81 and 88 , respectively). Due to small number of female recipients $(n=14)$, this analysis was not done separately for female gender.

\subsection{Predicting Acute Rejection and Post-Transplant Anti- HLA Antibodies Using Leptin}

The predictive power of serum leptin in acute rejection was also analyzed, but no significant ability to predict acute rejection was found at any time-point considered. Pretransplant anti-HLA screening was positive in 9 patients $(22.5 \%)$ and in 12 patients $(30 \%)$ during the first year following $\mathrm{KTx}$; in 2 patients (5.0\%) they were detected before transplant only, in 5 patients (12.5\%) after transplant only, and in 7 patients $(17.5 \%)$ both before and after transplantation. No significant association between leptin levels and anti-HLA positivity was showed.

\section{Discussion}

To date, most of the research on leptin and ADPN has been focused on its association with metabolic and cardiovascular health. Up to know, no reported study has examined the clinical utility of these adipokines in the diagnosis of graft dysfunction after KTx. We studied the performance of leptin and ADPN levels in predicting DGF using ROC analysis and compared these results with the routinely used SCr. Leptin 
levels at day-1, but not ADPN, slightly outperformed the traditional biomarker $\mathrm{SCr}$ in assessing the prognosis of DGF. Contrary to our expectations, leptinemia did not add substantially to the early discriminative or predictive power of $\mathrm{SCr}$ for the detection of DGF. Although degradation pathways of leptin have not been clearly elucidated, it has been suggested that endogenous leptin is rapidly cleared from circulation by glomerular filtration and by renal uptake and degradation [6,25-27]. However, it is possible that other factors that coexist in the immediate post-transplant period might stimulate leptin synthesis and delay its elimination.

Inflammation [28] has been implicated in augmenting leptin secretion. Surgical stress is also associated with an increase in serum leptin concentration $[29,30]$. Both of these conditions concur in KTx. Kidney transplantation is a surgical procedure that involves an inevitable ischemia-reperfusion injury with consequent deleterious activation of cellular oxidases causing oxidative damage, tissue injury and inflammation [31]. Leptin is an acute phase reactant that is involved in the cytokine network of acute inflammation and stress response [29]. It is possible that inflammatory cytokines resulting from the organ transplant process can stimulate leptin synthesis and attenuate its clearance from circulation, mainly in recipients with graft dysfunction. This would also explain why the decrement of plasma leptin concentration in the immediate post-transplant period did not reach normal levels in most of patients, even in those with prompt graft function. At day-7, $64 \%$ of our recipients still had levels that exceeded the upper limit of leptin reference range, or $40 \%$ considering only the recipients with prompt graft function. Some other studies show the maintenance of elevated serum concentrations of leptin in KTx recipients [32,33].

This study was designed based upon the assumption that impaired clearance of leptin (and ADPN) could signal graft dysfunction earlier than SCr. Of the two adipokines measured, leptin most closely fulfilled our initial hypothesis. Adiponectinemia was not significantly higher in recipients with graft dysfunction and was not a predictor of DGF. At least during the first week, graft dysfunction did not reflect impaired clearance of ADPN, suggesting that factors other than renal function may be involved. A study from Song and coworkers [34] demonstrated a decline in circulating ADPN levels during the initial $72 \mathrm{~h}$ after a subtotal nephrectomy in mice with renal failure, associated to down regulation of ADPN. Following this reasoning, we can also speculate that the decrease of circulating ADPN levels observed within the first week after KTx could be due to two different mechanisms according to graft function: enhanced filtration of circulating ADPN and urinary excretion in prompt graft patients; and decline in local expression of ADPN in glomerular endothelium as a result of amplified ischemia-reperfusion injury that usually describes DGF.

Ischemia/reperfusion injury is undoubtedly an important variable that can influence the outcome of the transplanted kidney since it is a major risk factor for the development of DGF and acute rejection [35,36]. In fact, there is an apparent synergy between the initial injuries of ischemia/reperfusion and acute graft rejection [37], and because of innate immune response this deleterious condition can lead to graft dysfunction [38]. Hence, the immune response against a transplanted organ may not solely involve a major histocompatibility complex specific alloimmune response, but in addition, an immediate nonspecific inflammatory response caused by ischemia/reperfusion injury [39]. Recent studies highlight the role of leptin in the immune system [9,20-24], therefore and beyond DGF we assessed leptin ability to predict acute rejection and anti-HLA antibodies following KTx. Possibly because of the small number of patients with acute rejection and anti-HLA antibodies no significant predictive value was found. Few studies [22] have addressed the influence of leptin, or other adipose tissue-derived products, on the allograft response and outcome, and to the best of our knowledge none in KTx.

In summary, the findings from the present study clearly demonstrate the importance of graft function in the clearance of leptin from the circulation, but not that of ADPN. Graft function was a stronger determinant of leptinemia, and the levels of this adipokine slightly outperformed $\mathrm{SCr}$ in predicting DGF. The maintenance of elevated levels of leptin in KTx and the role of this adipokine in allo-immunity are some of the questions that arise from this study, showing that much is still unknown in this field [40].

\section{Funding Sources}

None.

\section{Declaration of Interest}

The authors declare no conflict of interest regarding the publication of this article.

\section{Appendix A. Supplementary data}

Supplementary data to this article can be found online at http://dx.doi.org/10.1016/j.metabol.2014.10.003.

\section{REFERENCES}

[1] Sahin-Efe A, Katsikeris F, Mantzoros CS. Advances in adipokines. Metabolism 2012;61:1659-65.

[2] Wannamethee SG, Tchernova J, Whincup P, Lowe GD, Kelly A, Rumley A, et al. Plasma leptin: associations with metabolic, inflammatory and haemostatic risk factors for cardiovascular disease. Atherosclerosis 2007;191:418-26.

[3] Nakamura K, Fuster JJ, Walsh K. Adipokines: a link between obesity and cardiovascular disease. J Cardiol 2014; 63:250-9.

[4] Van de Voorde J, Pauwels B, Boydens C, Decaluwe K. Adipocytokines in relation to cardiovascular disease. Metabolism 2013;62:1513-21.

[5] Becker B, Kronenberg F, Kielstein JT, Haller H, Morath C, Ritz $\mathrm{E}$, et al. Renal insulin resistance syndrome, adiponectin and cardiovascular events in patients with kidney disease: the mild and moderate kidney disease study. J Am Soc Nephrol 2005;16:1091-8. 
[6] Cumin F, Baum HP, Levens N. Leptin is cleared from the circulation primarily by the kidney. Int J Obes Relat Metab Disord 1996;20:1120-6.

[7] Kataoka H, Sharma K. Renal handling of adipokines. Contrib Nephrol 2006;151:91-105.

[8] Komura N, Kihara S, Sonoda M, Maeda N, Tochino Y, Funahashi $\mathrm{T}$, et al. Increment and impairment of adiponectin in renal failure. Cardiovasc Res 2010;86:471-7.

[9] Moraes-Vieira PM, Bassi EJ, Araujo RC, Camara NO. Leptin as a link between the immune system and kidney-related diseases: leading actor or just a coadjuvant? Obes Rev 2012; 13:733-43.

[10] Saraheimo M, Forsblom C, Fagerudd J, Teppo AM, PetterssonFernholm K, Frystyk J, et al. Serum adiponectin is increased in type 1 diabetic patients with nephropathy. Diabetes Care 2005;28:1410-4.

[11] Shoji T, Nishizawa Y, Emoto M, Maekawa K, Hiura Y, Tanaka $\mathrm{S}$, et al. Renal function and insulin resistance as determinants of plasma leptin levels in patients with NIDDM. Diabetologia 1997;40:676-9.

[12] Zoccali C, Mallamaci F, Tripepi G. Adipose tissue as a source of inflammatory cytokines in health and disease: focus on end-stage renal disease. Kidney Int Suppl 2003:S65-8.

[13] Ziegelmeier M, Bachmann A, Seeger J, Lossner U, Kratzsch J, Bluher $\mathrm{M}$, et al. Adipokines influencing metabolic and cardiovascular disease are differentially regulated in maintenance hemodialysis. Metabolism 2008;57:1414-21.

[14] Chudek J, Adamczak M, Karkoszka H, Budzinski G, Ignacy W, Funahashi $\mathrm{T}$, et al. Plasma adiponectin concentration before and after successful kidney transplantation. Transplant Proc 2003;35:2186-9.

[15] Kokot F, Adamczak M, Wiecek A. Plasma leptin concentration in kidney transplant patients during the early posttransplant period. Nephrol Dial Transplant 1998;13:2276-80.

[16] Landt M, Brennan DC, Parvin CA, Flavin KS, Dagogo-Jack S, Coyne DW. Hyperleptinaemia of end-stage renal disease is corrected by renal transplantation. Nephrol Dial Transplant 1998;13:2271-5.

[17] Kayacan SM, Yildiz A, Kazancioglu R, Sahin S, Sever MS, Ark E. The changes in serum leptin, body fat mass and insulin resistance after renal transplantation. Clin Transplant 2003; 17:63-8.

[18] El Haggan W, Chauveau P, Barthe N, Merville P, Potaux L, Aparicio M. Serum leptin, body fat, and nutritional markers during the six months post-kidney transplantation. Metabolism 2004;53:614-9.

[19] Souza GC, Costa C, Scalco R, Goncalves LF, Manfro RC. Serum leptin, insulin resistance, and body fat after renal transplantation. J Ren Nutr 2008;18:479-88.

[20] Paz-Filho G, Mastronardi C, Franco CB, Wang KB, Wong ML, Licinio J. Leptin: molecular mechanisms, systemic pro-inflammatory effects, and clinical implications. Arq Bras Endocrinol Metabol 2012;56:597-607.

[21] Conde J, Scotece M, Gomez R, Lopez V, Gomez-Reino JJ, Lago F, et al. Adipokines: biofactors from white adipose tissue. A complex hub among inflammation, metabolism, and immunity. Biofactors 2011;37:413-20.

[22] Moraes-Vieira PM, Bassi EJ, Larocca RA, Castoldi A, Burghos $\mathrm{M}$, Lepique AP, et al. Leptin deficiency modulates allograft survival by favoring a Th2 and a regulatory immune profile. Am J Transplant 2013;13:36-44 [corrected].

[23] Moraes-Vieira PM, Larocca RA, Bassi EJ, Peron JP, AndradeOliveira V, Wasinski F, et al. Leptin deficiency impairs maturation of dendritic cells and enhances induction of regulatory T and Th17 cells. Eur J Immunol 2014;44:794-806.

[24] De Rosa V, Procaccini C, Cali G, Pirozzi G, Fontana S, Zappacosta S, et al. A key role of leptin in the control of regulatory $\mathrm{T}$ cell proliferation. Immunity 2007;26:241-55.

[25] Meyer C, Robson D, Rackovsky N, Nadkarni V, Gerich J. Role of the kidney in human leptin metabolism. Am J Physiol 1997; 273:E903-7.

[26] Cumin F, Baum HP, Levens N. Mechanism of leptin removal from the circulation by the kidney. J Endocrinol 1997;155: 577-85.

[27] Zeng J, Patterson BW, Klein S, Martin DR, Dagogo-Jack S, Kohrt WM, et al. Whole body leptin kinetics and renal metabolism in vivo. Am J Physiol 1997;273:E1102-6.

[28] Maury E, Brichard SM. Adipokine dysregulation, adipose tissue inflammation and metabolic syndrome. Mol Cell Endocrinol 2010;314:1-16.

[29] Chachkhiani I, Gurlich R, Maruna P, Frasko R, Lindner J. The postoperative stress response and its reflection in cytokine network and leptin plasma levels. Physiol Res 2005;54:279-85.

[30] Hernandez C, Simo R, Chacon P, Sabin P, Baena JA, Castellanos JM, et al. Influence of surgical stress and parenteral nutrition on serum leptin concentration. Clin Nutr 2000;19:61-4.

[31] Eltzschig HK, Eckle T. Ischemia and reperfusion-from mechanism to translation. Nat Med 2011;17:1391-401.

[32] Kokot F, Adamczak M, Wiecedil A, Spiechowicz U, Mesjasz J. Plasma immunoreactive leptin and neuropeptide $Y$ levels in kidney transplant patients. Am J Nephrol 1999;19: 28-33.

[33] Kagan A, Haran N, Leschinsky L, Sarafian R, Aravot D, Dolberg J, et al. Serum concentrations of leptin in heart, liver and kidney transplant recipients. Isr Med Assoc J 2002;4:213-7.

[34] Song Y, Yu Q Zhang J, Huang W, Liu Y, Pei H, et al. Increased myocardial ischemia-reperfusion injury in renal failure involves cardiac adiponectin signal deficiency. Am J Physiol Endocrinol Metab 2014;306:E1055-64.

[35] Kosieradzki M, Rowinski W. Ischemia/reperfusion injury in kidney transplantation: mechanisms and prevention. Transplant Proc 2008;40:3279-88.

[36] Ponticelli C. Ischaemia-reperfusion injury: a major protagonist in kidney transplantation. Nephrol Dial Transplant 2014;29(6):1134-40.

[37] Dragun D, Lukitsch I, Tullius SG, Qun Y, Park JK, Schneider W, et al. Inhibition of intercellular adhesion molecule-1 with antisense deoxynucleotides prolongs renal isograft survival in the rat. Kidney Int 1998;54:2113-22.

[38] Li L, Okusa MD. Macrophages, dendritic cells, and kidney ischemia-reperfusion injury. Semin Nephrol 2010;30:268-77.

[39] Koo DD, Welsh KI, Roake JA, Morris PJ, Fuggle SV. Ischemia/ reperfusion injury in human kidney transplantation: an immunohistochemical analysis of changes after reperfusion. Am J Pathol 1998;153:557-66.

[40] Modan-Moses D, Paret G. Leptin and transplantation: pieces are still missing in the puzzle. Isr Med Assoc J 2002;4:207-8. 